\title{
An interview with Antônio Carlos de Oliveira Ruellas
}

"Associate professor of Orthodontics, School of Dentistry - Federal University of Rio de Janeiro (UFRJ).

" MSc and PhD in Dentistry (Orthodontics), School of Dentistry - UFRJ.

» Former Professor at Federal University of Alfenas (Unifal) and University of Alfenas (Unifenas).

» Post-Doc in Materials Sciences at the Military Institute of Engineering (IME).

With joy and trust, I have accepted the invitation to coordinate this interview with professor Dr. Antônio Carlos de Oliveira Ruellas, one of the most renowned men in Brazilian Orthodontics. For those who do not know him, he is from the town of Areado (near the cities of Alfenas and Poços de Caldas) in the state of Minas Gerais. In 1989, he got a degree in Dentistry (School of Pharmacy and Dentistry - Alfenas - EFOA/Unifal) and two years later, under the advice of professor Walter Alves Araújo, he began his career as a professor of Dentistry at the University of Alfenas/Unifenas while he was still in the undergraduate course. Later on, professor Ruellas taught Physics at a university entrance exam preparation course in Alfenas. Subsequently, he went for the masters and doctorate degree in Orthodontics at the Federal University of Rio de Janeiro (UFRJ) where he stood out as one of the best students and, later on, one of the professors of that renowned institution - a position he has skillfully occupied until these days. Professor Ruellas has recently finished his post-doc in Engineering of Metal and Materials at the Military Institute of Engineering (IME). He has published more than 190 articles in national and international periodicals, in addition to two books (his most recent books is entitled "Biomechanics applied to the clinical practice", a masterpiece of the orthodontic literature), among others. Professor Ruellas stands out from the crowd not only for his titles, academic production and attributes, but even more for his simplicity, humbleness and generosity towards those around him. He has always supported his students to go beyond him by saying "My students should be better than I am, and their students should be better than what they are...". At work, he passes on everything he knows, and when he does not know something, he admits it, always seeking more and more knowledge. I consider professor Antônio Carlos as my orthodontic father - I dived into Orthodontics because of his advice and his lessons. I had the privilege of being his student during the undergraduate, postgraduate, masters and doctorate courses. And that makes me very proud. I do not know anyone else who has had the privilege of being his student during all learning stages. I would like to end this preface by inviting everyone to read and enjoy this interview that will certainly expand our orthodontic knowledge.

Matheus Melo Pithon

" Patients displayed in this interview previously approved the use of their facial and intraoral photographs.

How to cite this interview: Ruellas ACO. Interview. Dental Press J Orthod. 2013 Nov-Dec;18(6):15-25.

Submitted: August 07, 2013 - Revised and accepted: September 05, 2013 
With regard to vertical Class II skeletal malocclusion treatment during growth, which are the therapeutic options? What are the possibilities of employing skeletal anchorage to control growth in these cases? Is there any scientific evidence and what are the future prospects?

(André Weissheimer)

A successful treatment for a vertical Class II skeletal malocclusion depends on precisely controlling growth direction. I believe that considering whether or not growth direction is predominant due to some habits, such as mouth breathing, plays a major role both in diagnosis and prognosis. This is normally present due to difficulty in lip seal caused by proclination of the upper incisors. Controlling the vertical alveolar growth, especially in the maxilla, will enable mandibular counterclockwise rotation.

When deleterious habits are present, their removal is enough to recover growth direction, at least partially. Additionally, employing mechanics to control vertical alveolar growth will yield satisfactory results. The mechanics that can be used to control vertical alveolar growth are: High-pull headgear, vertical chin cup, mini implant (MI) and miniplates. High-pull headgears, MIs and miniplates require that the applied force be transmitted to the posterior teeth by means of a rigid arch, and that it does not remain restricted to one or two posterior teeth, only. Although being effective, high-pull headgears and vertical chin cups (especially the high-pull headgear) totally depend on patients' cooperation. Nowadays, this requires that the orthodontist be highly persuasive and able to show to the patient not only the importance of using the appliances, but also their consequent benefits. Due to being increasingly rejected by patients, the use of high-pull headgears and vertical chin cups has significantly decreased.

Conversely, MIs and miniplates do not require patients' cooperation (except for the need of specific hygiene for that area). The miniplates probably yield more satisfactory results due to the possibility of providing support outside the alveolar bone area. However, both require the need for surgery, especially the miniplate (for which the intervention of another professional is necessary), which implies higher costs.

The future prospects are that temporary skeletal anchorage will be more used, as costs tend to decrease, stability tends to increase, patients tend to become more aware and familiarized with this type of therapeutic resource and, as a consequence, tend to reject it less.

Although many researches have assessed the use of skeletal anchorage for the Class III treatment, ${ }^{1-5}$ additional studies are warranted to further investigate its use for Class II treatment. It is possible that, in a very near future, new findings will be published about this topic.

Treatment of skeletal Class II malocclusion is often associated with tooth extraction in only one or in both arches. What are the requirements that should be taken into account to perform tooth extraction in one or in both arches?

(Matheus Melo Pithon)

Extraction performed in the upper arch is recommended for Class II patients whose mandibular arch is in good conditions (positive discrepancy, little or no negative discrepancy) or in cases of proclination in the mandibular arch, in which the mandibular incisors will be compensated, since repositioning them would lead to overjet, which is normally significant, and for this reason, hinders correction. Thus, extractions in the upper and lower arches are recommended in the following situations:

" Negative lower discrepancy (cephalometric discrepancy + dental discrepancy), from moderate to accentuated value; or mild when associated with one of the factors described below (protruded profile, intensified vertical dimension, lack of lip seal). Extractions allow the creation of space for alignment and leveling of teeth, in addition to favoring passive lip seal due to correction of incisor protrusion.

" Protruded profile: Since facial esthetics is highly valued, extractions should be considered to improve labial protrusion and facial profile esthetics. Should extractions be recommended for other reasons (such as little total discrepancy) or should they worsen facial esthetics, they must be questioned and discussed. Patient's age and ethnicity may also be taken into account. Changes in the facial profile of adult patients may age them; therefore, such changes must be carefully performed. One should also seriously consider obtaining a slightly convex profile for adolescents, since their profile tend to become straight and/or concave with age. 
» Increased vertical dimension: In this case, extraction of lower second premolars may be considered to obtain vertical control and molar occlusion relationship, which would facilitate anchorage loss, especially if mandibular arch discrepancy is mild and changes in facial profile are not absolutely necessary. However, the patient must be advised not to apply excessive force during oral hygiene in order to avoid potentiating recession in the lower first molar mesiobuccal root. Moreover, with this same purpose, the clinician must promote an adequate torque control and lower molar offset for the mesially moving molar (excessive buccal root torque and offset may potentiate gingival recession). Thus, the arch must be adjusted with compensating bends (or by modifying those that already exist).

In my opinion, mass retraction should be carried out in cases in which anteroposterior and vertical anchorage control is not compromised. In other words, during retraction of anterior teeth, both mesial migration of posterior teeth and increase in maxillary incisor exposure at rest are allowed. As a result, the maxillary incisor would be more exposed in relation to the lip, and overbite would increase. Thus, prior to treatment onset, the patient should have slight lip protrusion in relation to the face, little overbite as well as little exposure of the maxillary incisor. However, in most cases, patients do not conform to these features and if they choose to perform mass retraction, both anteroposterior and vertical mechanical control may be compromised and result in gingival smile and increased overbite. The best alternative to perform mass retraction in these situations is doing so by means of mini-implants ${ }^{6}$ (Fig 1). A hook is welded to the canine distocervical wing of enough height to allow the elastomeric chain, which pulls the tooth against the mini-implant, to be horizontally placed. This results in canine distal movement and minor intrusion of the upper incisors. The more tipped the elastomeric chain (in the same direction as shown in Fig 1), the greater the incisor intrusion. From the second appointment on to activate the space closure system and replace the elastomeric chains, the teardrop loop is activated by anchoring the distal hook to the loop up to the MI.

Although retraction of canines and incisors will be simultaneously performed with different mechanical controls, they complement each other because incisor

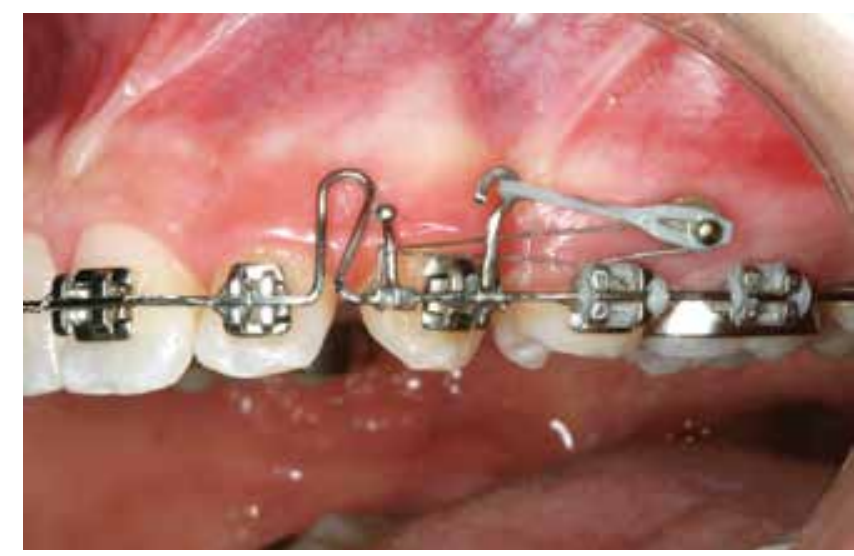

Figure 1 - Photograph showing mass retraction supported by mini-implants.

intrusion, happening as a result of an appropriately placed canine hook, helps with vertical control of anterior teeth during retraction. The vertical effect (extrusion) of posterior teeth may be suppressed by a steel wire ligature placed from the MI to the arch and anchored between the premolar and the first molar. As for the control of buccolingual tipping of anterior teeth, it is performed by means of torquing bends, if necessary.

With regard to treatment of dental Class II malocclusion in adult patients, which are the aspects that should be considered when opting for treatments that comprise extraction of upper first premolars or skeletal anchorage used for distalization? What are the recommendations, limitations, advantages and disadvantages of each treatment option? (André Weissheimer)

Dental Class II malocclusion in adult patients comprises the following options of orthodontic treatment:

a) Distal movement of upper teeth, which may be performed with headgear appliance, intraoral jig appliances, intermaxillary elastics with Class II direction, MIs or miniplates.

b) Upper premolar extractions (PM).

The choice between these two options should be based on the following:

a) Amount of distalization: The greater the need for distalization, the stronger the decision leans towards extraction of PM. In my opinion, distalization must not be greater than $3 \mathrm{~mm}$, i.e., not greater than half of the PM mesiodistal width. Except for cases in which the vertical dimension of the facial lower third has decreased. 
b) Vertical dimension (VD): Increased VD, difficulty with lip seal, increased lower facial third and decreased overbite are some of the aspects one should take into account when planning treatment that involves extraction of upper PM. The same aspects, but in opposite conditions, tend to direct treatment towards distalization.

c) Presence of third molars: In the presence of fully formed third molars, I tend to recommend extraction of PM because it is probable that, as a result, there will be enough space for eruption of the third molar - which, without extraction of PM, probably would not occur. The patient would have the same number of teeth in the oral cavity and both of them are dental organs. Treatment would be quicker and would involve fewer risks (there would be little need for tooth movement). Additionally, the chances of replanning and changing the procedures would decrease (should distalization be ineffective or the MI be unstable). In cases in which distalization is considered, I usually recommend extraction of the third molar, depending on its position and the space available in the posterior region (given that two bodies cannot occupy the same space at once).

d) Maxillary tuberosity bone: When distal movement is planned, it is necessary to assess the amount of bone present in the maxillary tuberosity. Is there bone so that teeth may be distally moved? Should there not be enough bone, I recommend extraction of PM.

e) Cooperation: Adequately cleaning the MI is essential to maintain its stability and may influence on the treatment success performed with distalization supported by the MIs. This aspect will be even more critical if the treatment choice involves a headgear appliance or intermaxillary elastics with Class II direction.

f) Second molar tipping: At first, during eruption, this tooth is distally tipped. This tipping is corrected as the tooth erupts and the tuberosity grows. Distal tipping of second molars usually represents lack of space in the posterior region (lack of bone or presence of the third molar which hinders uprighting of the second molar). Thus, extraction of upper PMs is recommended.

g) Retreatment: This planning must be objective and precise. Therefore, if distalization has already been attempted and yielded unsuccessful results, I suggest that this procedure be avoided.
These items work as guides, since assessment does not depend on the number of pros or cons against a decision, only, but on the importance each item has over the decision of planning each case individually. It is worth noting that professional clinical experience is of paramount importance for all kinds of orthodontic planning, since it allows the professional to easily visualize solutions and identify alternatives. That is the reason why I usually assert that all health professionals should go through a training period (in my opinion, for five years) after they finish their specialization course, or that they had a "tutor" (someone with more experience, who would work as their "advisor", helping them on their planning). Well-structured courses and committed students lead to excellent technicalscientific preparation of recently graduated professionals, but clinical experience cannot be totally passed on.

Class II malocclusion at the end of the mixed dentition, near pubertal growth spurt, is traditionally treated with headgear appliances. Do you believe it is possible to treat Class II malocclusion with mini-implants? Are you aware of any scientific research by which both methods are compared? (Márlio Vinícius de Oliveira)

Studies assessing treatment of Class III performed with skeletal anchorage have been well reported in previous articles. ${ }^{2,7}$ However, the same has not happened with Class II treatment. I do not believe that miniimplants can be considered an appropriate anchorage procedure employed to control anteroposterior growth in the Class II treatment. I believe that miniplates are more successful in the Class II treatment, especially in the vertical control of alveolar growth, given that it allows mandibular counterclockwise rotation and, as a result, better spacial positioning in the anteroposterior direction, which decreases the ANB angle and improves facial profile esthetics. As for the mini-implants, ${ }^{8-13}$ I believe they are limited in working as a support for distal movements or as anchorage in cases of premolar extractions for, correcting dental Class II or compensating skeletal Class II malocclusions. I consider that even severe Class II corrections with good facial growth pattern can be easily obtained with functional appliances (high-pull headgear, Herbst appliance) provided that their indications and characteristics have been correctly examined. The biggest problem consists 
in correcting Class II malocclusions with adverse growth pattern (vertical displacement), in which case the MIs ${ }^{14}$ and especially the miniplates will yield good results, thus, substituting the extraoral appliances (currently, the best tool that provides the best mechanical control in these cases).

With regard to Class II treatment, what is the age limit to use fixed functional appliances, such as the Herbst and others?

(Luiz Gonzaga Gandini Júnior)

Functional appliances to control bone displacement from facial growth, and consequently correct a Class II, are mostly recommended during the growth spurt phase. Thus, they would be recommended for girls not older than 13 years old, and for boys not older than 14 years old. Using these appliances at a subsequent age will produce mainly dental effects, with correction by compensation and stability that depends on the patient's initial features (lower incisor buccolingual tipping, mandibular plane tipping, vertical dimension, lip seal, mandibular muscle tone). One should also consider potential effects of fixed functional appliances on TMJ (especially on the retrodiscal ligaments, articular disc and capsule), mainly in adult patients whose capacity of adaptation is limited. Some researchers have assessed the effects of using the Herbst appliance in adults, ${ }^{21-24}$ and have concluded that dental effects tend to be more prevalent as the patient gets older: ${ }^{21}$ In my opinion, it should not be used in adult patients and treatment should not be finished with the mandible more anteriorly positioned in relation to the articular cavity. ${ }^{25}$

Given the great diversity of biomechanical systems, which force system do you prefer to use to upright mesially tipped lower molars in cases of space closure (mesial movement of the root) - with and without vertical control, taking into account simplicity, patient's comfort, clinical efficiency and predictability of results?

(André Weissheimer)

I believe that the best system used to upright lower molars that have been tipped due to loss of mesially positioned teeth comprises MIs, especially when the MIs can be distally positioned in relation to the molar that will be uprighted. ${ }^{6}$ Such position allows better vertical control of molars, but is recommended in cas- es in which space will be opened through distal movement of the crown and posterior rehabilitation of the edentulous space. Uprighting through mesial movement of the root is more difficult to be performed due to the great amount of bone that will have to be reabsorbed in the region mesial to the root. Mesial movement of the root without vertical control can be achieved with an uprighting spring with the support of a segmented arch or a MI (Figs 2A and B). Absence of vertical control is sometimes recommended, especially when the purpose is to gain vertical dimension of the lower facial third for later rehabilitation. It is worth noting that it is necessary to keep adequate free functional space. Vertical control should be associated with some other tool in order to intrude the molar during uprighting, thus, preventing the molar crown from occupying a more "extruded" position.

As for uprighting of molars, what are the parameters used to choose between segmented mechanics and straight archwires? When are Temporary Anchorage Devices (TAD) used, what is the determining factor of such a choice?

\section{(Luiz Gonzaga Gandini Júnior)}

I consider the vertical aspect as one of the determining factors when choosing between segmented mechanics and straight archwires to upright lower molars. That is because straight archwires allow greater extrusion of the mid segment of the arch in comparison to segmented arches. Additionally, controlling the buccolingual position of the mesially positioned tooth towards the edentulous space is safer when performed with segmented arches. In general, the second premolar must be leveled to the mesially positioned dentition by an rigid arch. Thus, I believe that in most cases (except for mild tippings) the segmented arch mechanics should be used for this purpose. In cases of space closure, I suggest that - should there be any space left to be closed after complete uprighting carried out through mesial movement of the root - it be performed with straight archwire, from this point on.

I recommend that uprighting be, as much as possible, supported by TAD, only (with the segmented arch going from the TAD to the tooth that will be uprighted), in order to prevent side effects on the dentition (extrusion, transversal movements, anteroposterior movements). The greater the initial tipping of the 

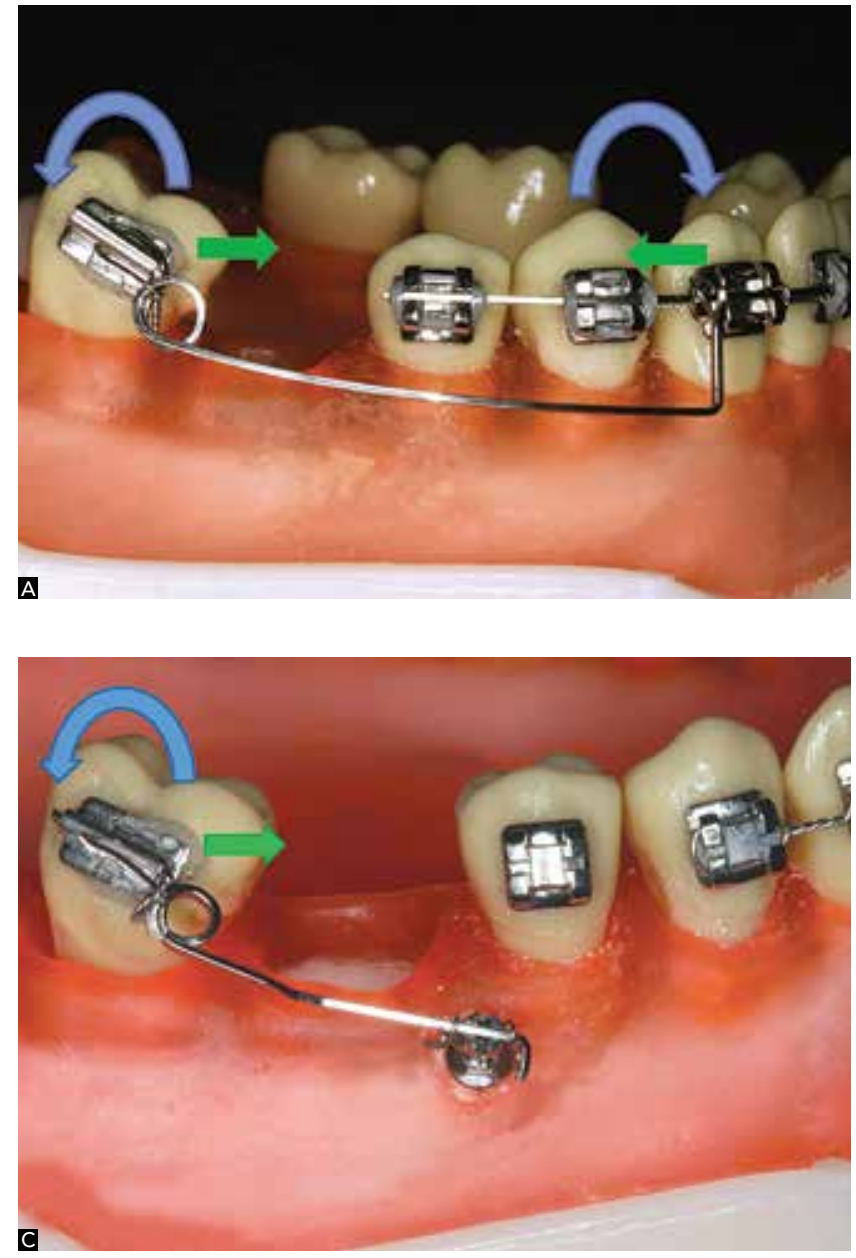
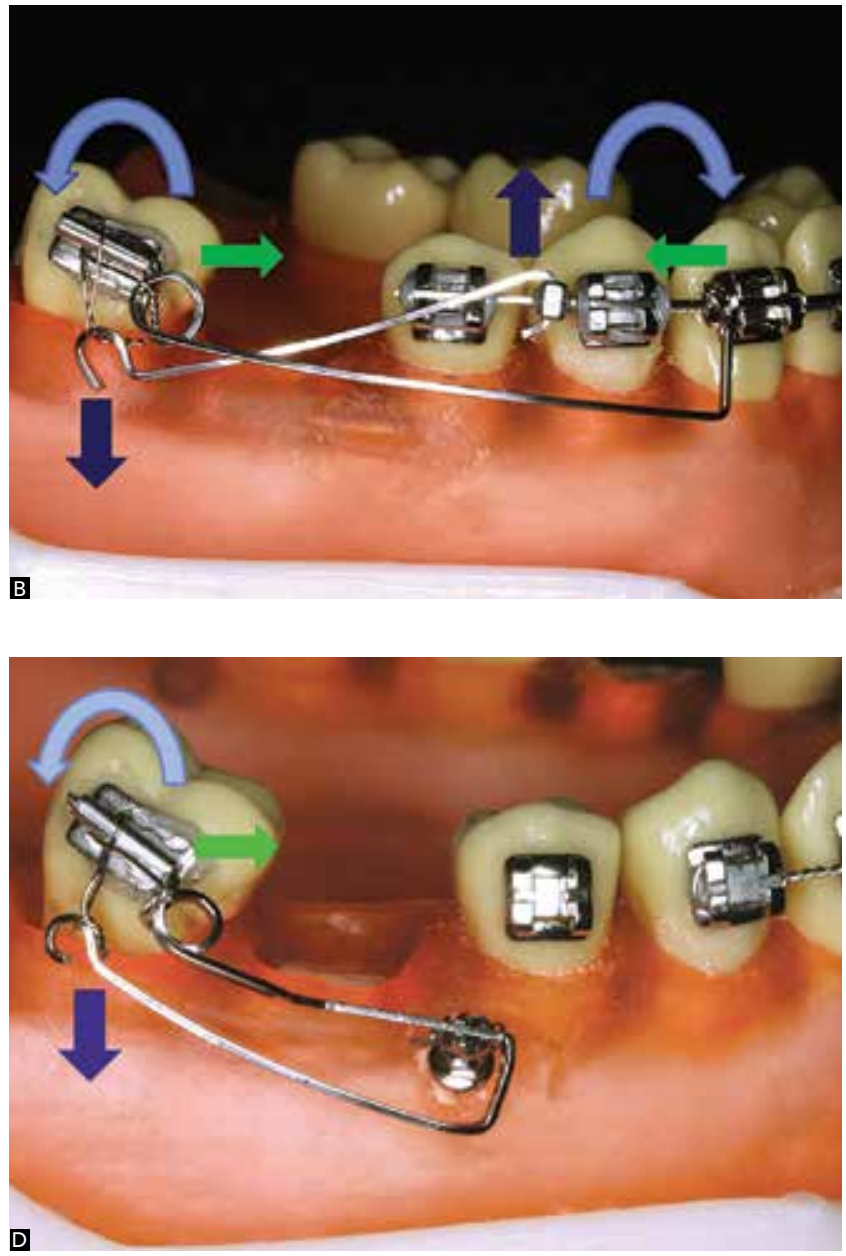

D

Figure 2 - Molar uprighting through mesial movement of the root, without vertical control. A, B) without MI support; C, D) with MI support; A, C) without vertical control; B, D) with vertical control. The uprighting spring must have its helicoidal surface attached to the tube in all options. In A and B, the mesial portion of the spring, which is fitted between the premolar and the canine, is mesially inserted into the bracket of the canine; avoiding distal movement of the molar crown. In C and D, the mesial portion of the spring is fitted in the MI and must end close to the MI, avoiding distal movement of the molar crown. After the molar has been uprighted, I recommend that the spring be replaced by a loopless straight archwire and that the molar be mesially pulled (especially by buccolingual inclination, avoiding rotation) with mild force, in a $0.018 \times 0.025$-in archwire, avoiding new mesial inclination, with or without MI support.

molar that will be uprighted and the space that needs to be closed (if applicable), the greater the need to use TAD. MI is the most used tool for these cases. Should the MI be distally positioned towards the molar that will be uprighted, vertical control will be more effective. However, should it be mesially positioned, vertical control will be hindered. Depending on the clinical case, even if TAD is used, molar vertical control may not be as effective. Extrusion will be limited to the molar, only, in which case occlusal adjustments or rehab treatments may be performed (if necessary).

The current stage of development of mini-implants allows us to treat complex malocclusions which, not long time ago, were treated by means of orthognathic surgery, only. Have you used this mechanism to treat anterior open bite, for example? What is the mechanical planning (number and positioning of mini-implants) most widely used for this purpose?

\section{(Márlio Vinícius de Oliveira)}

MIs have considerably helped orthodontic treatments and, in many open bite cases, they may eliminate the need for orthognathic surgery, provided that the MIs present satisfactory stability during treatment. In some cases, it is possible to eliminate the need for orthognathic surgery by correcting the skeletal problem (especially when growth is still ongoing) or treatment by compensation (without correction of the skeletal problem, but by obtaining compensatory dental positions that disguise it). In other cases, the need for orthognathic surgery may be minimized: Involving only one arch, for example, whereas when MIs are not used, both arches are involved. 


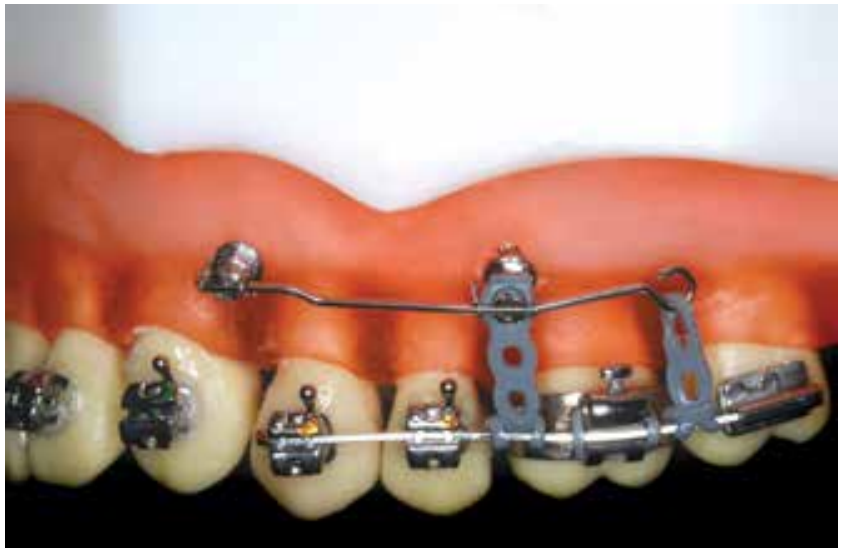

Figure 3 - Photograph showing intrusion of posterior teeth with MI support. ${ }^{6}$

Initially, I use the MIs in the arch where the posterior teeth have bigger clinical crowns (longer in the cervico-occlusal direction) and, afterwards, in the other arch, if necessary. For intrusion of posterior teeth in open bite correction, I use at least two mini-implants on each quadrant because either intrusion or vertical growth control will be necessary (depending on the patient's stage of development) in more than one tooth, thus, allowing greater support. Then, I apply elastomeric chains that go from the MIs to the rigid arch inserted into the brackets; or I use a segment of wire in both MIs (bracket head), which allows traction to be exerted in different points of the wire fixed to the brackets ${ }^{6}$ and in the segment of wire inserted into the MIs (Fig 3). In terms of position, I believe that a MI should be placed between premolars, while the other should be between first and second molars. However, due to difficulty in placing a MI between molars, I often place it between the premolar and molar. If the purpose is to achieve rotation of the occlusal plane (extrusion of incisors), straight wire mechanics may be used. If the effect in the incisors must be totally neutralized, intrusive force with the support of a segmented arch should be applied, acting on posterior teeth, only. After improvements in leveling the occlusal plane have been achieved, treatment begins to be performed with a straight wire.

It is worth noting that mechanics should be associated with recovery of patient's normal functions, especially nasal breathing and lip sealing, which are essential to reestablish normal growth conditions and correction stability.
Based on your professional experience, which factors are determining when choosing between intermaxillary elastics, tooth extraction, mini-implants, miniplates and orthognathic surgery to treat open bite?

(Luiz Gonzaga Gandini Júnior)

I believe that orthognathic surgery is restricted to two situations: Functional and/or esthetic limitation. In other words, if a key function cannot be obtained without surgery and/or if the patient is not satisfied with his facial esthetics due to the malocclusion, orthosurgical treatment is recommended. Otherwise, orthodontic treatment should be carried out, or at least tried to be performed. Miniplates and mini-implants are recommended to intrude posterior teeth (or to control vertical alveolar growth) and to reduce or control vertical dimension of the lower third of the face. The same applies to extractions, especially if the need for vertical control is associated with the need for space gain in the arch (distalization would lead to increased vertical dimension, and proclination of anterior teeth would lead to decreased overbite). Extractions with predominant mesial movement of posterior teeth can decrease vertical dimension. Thus, in case of extractions, we usually recommend extraction of second premolars, first or even second molars. Intermaxillary elastics are recommended for cases in which the vertical dimension is normal and the patient's features allow extrusion of incisors. That is to say, exposure of upper incisors at rest is decreased, or a minor increase in exposure of upper incisors, if normal, does not compromise facial esthetics and will not result in gingival smile. Such consideration regarding exposure of upper incisors must be taken into account according to patient's age, sex and upper lip length.

I believe that associating two or more of these procedures, whenever recommended and possible, is useful and will result in a more successful and stable correction. They are indispensable not only to recover muscle and respiratory functions (lips, tongue and muscles of mastication), but also for correction and stability. I also believe that associating spurs or cribs to control lingual interference is beneficial.

In cases of deep overbite, what are the parameters you choose when opting between anterior intrusion, posterior extrusion or a combination 
of both? In terms of mechanics, how do you work in each situation?

(Luiz Gonzaga Gandini Júnior)

At first, diagnosis is carried out by measuring the facial thirds. Should the lower third of the face have been decreased, deep overbite must be corrected by extruding posterior teeth. To diagnose the necessary amount of extrusion, the dentist places a wax roller between the occlusal surface of the posterior teeth, asking the patient to press it firmly until the facial thirds are proportional (measurements can be carried out by means of a pachymeter or a compass). The amount of interposed wax corresponds to the amount of necessary extrusion (Fig 4).

If the lower third of the face is proportional or if the deep overbite could not be totally corrected even after the interposed wax had been placed between the posterior teeth, complementary correction will be performed by intruding lower and/or upper anterior teeth. The need for intrusion of anterior superior teeth is examined by the amount of maxillary incisor exposure in relation to the upper lip at rest (half-open lips) and also by taking normal lip length into account (on average, $21 \mathrm{~mm}$ ). Normal exposure ranges from 2 to $4 \mathrm{~mm}$ for young men and from 3 to $5 \mathrm{~mm}$ for young women, and it decreases with age. Should exposure increase, intrusion of upper incisors must be carried out. Should exposure be normal or the upper incisor be normally exposed after intrusion, the lower incisors must be intruded. Intrusion of the upper incisor must be carefully carried out. Additionally, it is preferable that intrusion of the lower incisor be performed, especially in adult patients, since intrusion of upper incisors decreases exposure and ends up aging the patient when it is not correctly carried out.

Mechanically, the following options may be used to correct deep overbite:

a) Relative intrusion (relative extrusion) with straight wires, making use of mechanical procedures to avoid proclination of incisors, when it cannot occur.

b) Intrusion of incisors with Ricketts intrusion arch (when the first molar is mesially tipped, vertically positioned or retroclined, favorable growth pattern) or with Burstone intrusion arch (it allows better vertical control due to leveling by segment and stabilizing the posterior segments with segmented arch associated with palatal bar or lingual arch).

c) Extrusion of posterior teeth carried out by means of bite plate, fixed stops attached to the lingual surface of upper incisors, vertical elastics in the posterior region, accessory appliances (when necessary) which also potentiates posterior extrusion (cervical traction headgear, for example). Not only freeway space must be considered for extrusion of posterior teeth (after extrusion, at least $3 \mathrm{~mm}$ of freeway space must remain), but also some kind of physiotherapy that is performed to relax the elevator masticatory muscles and, thus, avoid potential relapse.
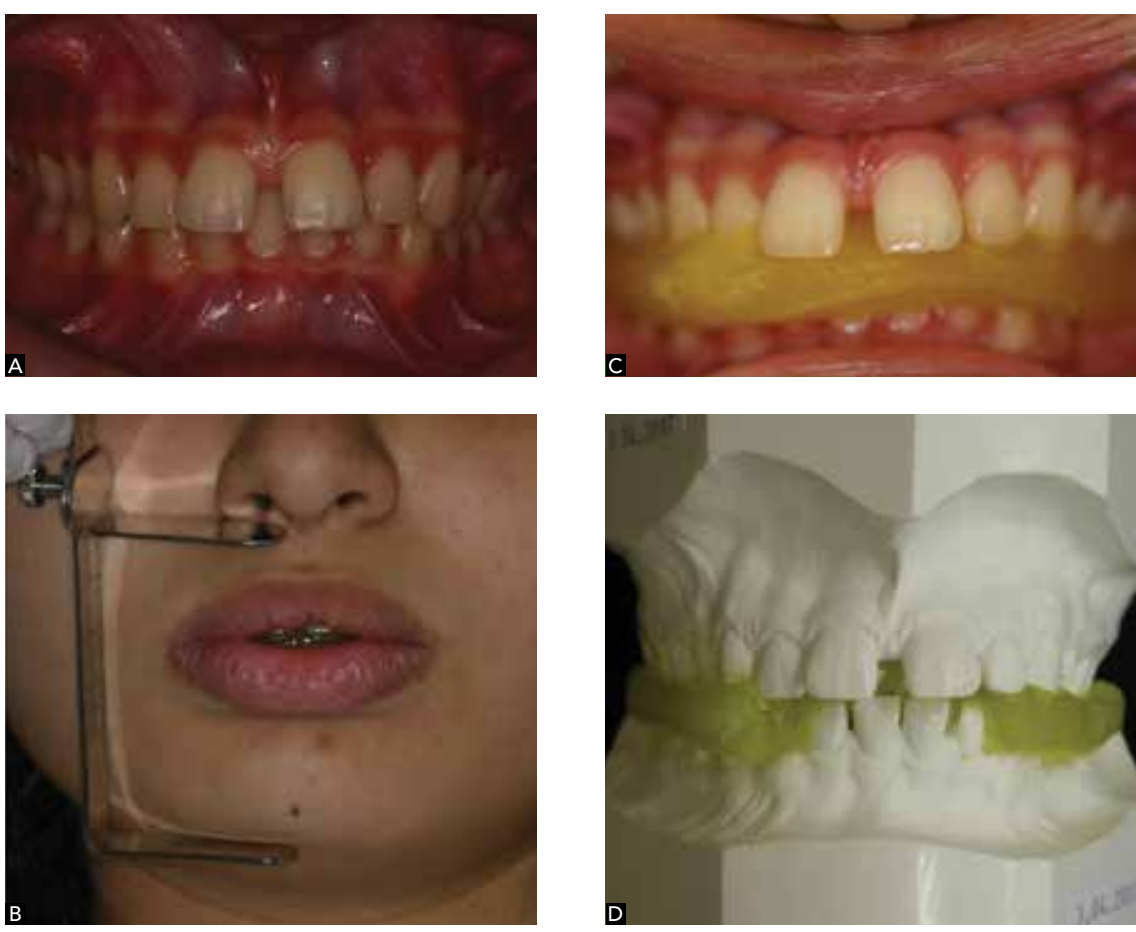

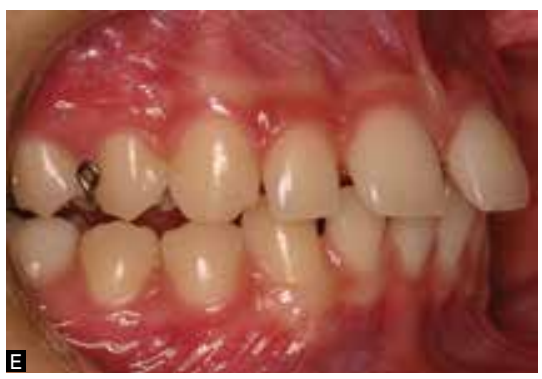

Figure 4 - Facial thirds proportion measured by means of interposing wax in the occlusion, and extrusion of posterior teeth obtained by means of a bite plate. 
As time goes by, orthodontic treatment performed in adult patients has increased exponentially. However, it is widely known that treatment performed in those patients requires considerable caution - especially due to dental absence, restored teeth or loss of periodontal support. From a biomechanical standpoint, which precautions must be considered when performing orthodontic treatment in adult patients?

(Matheus Melo Pithon)

Adult patients often present bone loss. In these cases, the center of resistance apically migrates, increasing its distance with regard to the location where force is applied (bracket). As a consequence, mechanical control in tipping movements will be hindered. A possible solution is to place the accessory in a more cervical direction in relation to the center of the clinical crown. Applying mild force will also help to prevent the arch from being deformed, especially in areas of extraction space, thus, minimizing tooth tipping. As for movement of incisors retraction, resistant torque usually needs to be increased for adequate control.

Due to greater bone density, application of mild force and a four-week interval between activations are recommended.

Frequent gingival recession, with little attached gingiva, also result in the need for careful mechanics - especially with regard to expansion or proclination movements, be it intentional or side effects of certain mechanics. For instance, when a spring is used for molar uprighting (Fig 2A), an intrusive effect will be generated in the upper premolars region, and this intrusive force will be inclined in relation to the center of resistance of these supporting teeth: Thus, they tend to be uprighted. Should a segmented arch be associated with it, from premolar to premolar, and present some expansion or inadequate torque, or should the uprighting spring not be contoured - following the curvature of the arch in an anterior direction - , premolars tend to expand laterally. In the region underlying the edentulous space, the presence of little attached gingiva is common, which may lead to total gingival loss if these variables are not controlled.

In order to obtain stability and occlusal balance in adult patients with many restorations, some occlusal adjustments and/or rehabilitation procedures are also necessary, since they adapt the occlusal condition to the new intercuspation that has been obtained.
Increase in the number of adult patients seeking orthodontic treatment leads to an increase in the demand for esthetic appliances. What is the stage of development of esthetic orthodontic archwires? Can they replace metal archwires in all stages of orthodontic treatment, even in more complex cases, such as those involving tooth extractions? (Márlio V. Oliveira)

There is no doubt that the esthetic aspect of orthodontic appliances has become a major concern in the last few years. Even though many options of esthetic archwires are available in the market, I believe they have not followed the development of ceramic brackets, with regard to appearance, color and esthetic layer stability or physical properties. The esthetic archwires currently produced are either archwires made of composite material (a polymer strengthened by glass fiber - fiber reinforced plastic, FRP, archwires) or metal archwires (made of steel or nickel-titanium, covered by a surface made of epoxy resin or polytetrafluoroethylene-based material, which is similar in color to the teeth). The FRP archwires are more esthetic, but cause more severe damage to the physical properties. Even though coated metal archwires present differences in physical properties (in comparison to non-coated archwires), ${ }^{15,16}$ they are not esthetically favorable. In other words, they do not "mingle" with the bracket and the tooth (they cannot be disguised). Additionally, they are not stable enough for maintenance of the coating material, ${ }^{17}$ which affects esthetics and may result in greater dental plaque accumulation.

Another important aspect worth noting is the friction in sliding mechanics, because the coating material has greater friction than metal, and when the coating material detaches, two problems may occur: Even greater friction due to irregularities caused by material detaching or due to irregularities caused by exposure of roughened metal archwire (some commercial brands have the metal surface treated so that the coating material can adhere to it more easily). This may compromise the characteristics related to: Corrosion, friction, esthetics, physical properties and colonization of microorganisms.

It is worth noting that esthetic archwires are under development - as it happened to other orthodontic materials in the past - and they will probably have good conditions for routine clinical application. 

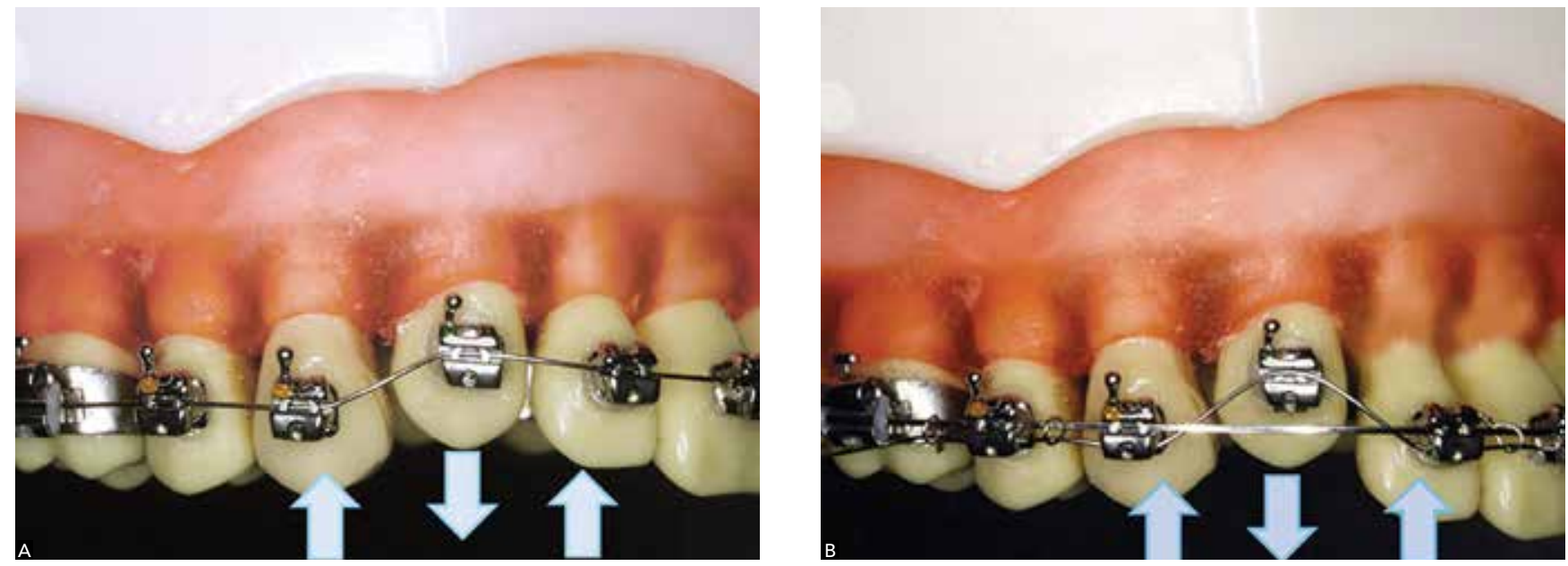

Figure 5 - Traction of tooth with self-ligating brackets. A) With NiTi archwire attached to all teeth. Deleterious effects on adjacent teeth (intrusion and buccal inclination, especially in the lateral incisor); B) procedure carried out to minimize deleterious effects on adjacent teeth supported by an rigid arch (the intrusive effects on adjacent teeth exist, but do not result in effect on the teeth due to the use of the rigid arch).

Thus, I have currently recommended them mainly for social events (for a weekend, for example) after which they must be replaced by metal archwires again. But I believe that not only patients, but also clinicians wait anxiously for their development and their better characteristics so that they can be clinically used.

Nowadays, we often hear about self-ligating appliances and their innumerable advantages. The materials industry and some researches give to this kind of device advantages such as decrease in discomfort after activation, less friction, shorter chair time and shorter treatment time. Based on your extensive experience in orthodontic biomechanics, biology of tooth movement and properties of orthodontic materials, I would like to ask you: Is it possible to gain so many benefits from these devices, simply due to the fact that they have a different mechanism that entraps the orthodontic archwire? (Matheus Melo Pithon)

I believe and trust in the new technologies that have been introduced into the orthodontic market. The technological development has been a remarkable characteristic of Orthodontics, not only in terms of diagnosis, but also in terms of treatment. Many technologies have been launched. Some of them have been forgotten, but other remained in the market and had their clinical applicability confirmed within biological limits. There is no doubt that self-ligating brackets have some significant advantages, especially with regard to easiness in bonding, which results in shorter chair time. However, tooth movement is a biological response to the application of force and the bracket functions as to transmit such force to the tooth. Thus, the side effects of some kind of mechanics will not be neutralized by the fact that the bracket is self-ligating, only. Discomfort, movement rate and treatment time will not be affected either, no matter the brand of the bracket (considering that all brackets are bonded to the dental surface during treatment and that the space between the archwire and the bracket is the same). High-quality accessories - with better finishing, which are resistant to corrosion and have more precise slot dimensions - certainly present better mechanical response and fewer risks of allergic reactions. However, being self-ligating will not transmit force to the tooth in a more or less favorable mechanical direction, with a higher or lower degree of force. Additionally, it will not neutralize the side effects of a mechanics that is used incorrectly (Fig 5). Some researches reveal no significant difference in terms of response to tooth movement when self-ligating brackets are compared to the conventional ones. ${ }^{18,19,20}$ 
1. Baccetti T, De Clerck HJ, Cevidanes LH, Franchi L. Morphometric analysis of treatment effects of bone-anchored maxillary protraction in growing Class III patients. Eur J Orthod. 2011;33(2):121-5

2. Cevidanes L, Baccetti T, Franchi L, McNamara JA, De Clerck H. Comparison of two protocols for maxillary protraction: bone anchors versus face mask with rapid maxillary expansion. Angle Orthod. 2010;80(5):799-806

3. De Clerck H, Cevidanes L, Baccetti T. Dentofacial effects of bone-anchored maxillary protraction: a controlled study of consecutively treated Class III patients. Am J Orthod Dentofacial Orthop. 2010;138(5):577-81.

4. Nguyen T, Cevidanes L, Cornelis MA, Heymann G, de Paula LK, De Clerck H. Three-dimensional assessment of maxillary changes associated with bone anchored maxillary protraction. Am J Orthod Dentofacial Orthop. 2011:140(6):790-8.

5. Lara JC, Facio Umaña JA. Modified miniplates for orthopaedic skeletal anchorage. Int J Oral Maxillofac Surg. 2012;41(5):566-8.

6. Ruellas ACO. Biomecânica aplicada à clínica. Maringá: Dental Press; 2013

7. De Clerck EE, Swennen GR. Success rate of miniplate anchorage for bone anchored maxillary protraction. Angle Orthod. 2011;81(6):1010-3.

8. Doshi UH, Jamwal RS, Bhad WA. Distalization of molars using two stage mini-implants: a case report. J Orthod. 2011;38(1):55-63.

9. Jung $\mathrm{MH}$. A comparison of second premolar extraction and miniimplant total arch distalization with interproximal stripping. Angle Orthod 2013;83(4):680-5.

10. Ueno S, Motoyoshi M, Mayahara K, Saito Y, Akiyama Y, Son S, et al. Analysis of a force system for upper molar distalization using a trans-palatal arch and mini-implant: a finite element analysis study. Eur J Orthod. 2013;35(5):628-33.

11. Upadhyay M, Yadav S, Nagaraj K, Nanda R. Dentoskeletal and soft tissue effects of mini-implants in Class II division 1 patients. Angle Orthod. 2009;79(2):240-7

12. Upadhyay M, Yadav S, Nagaraj K, Uribe F, Nanda R. Mini-implants vs fixed functional appliances for treatment of young adult Class II female patients: a prospective clinical trial. Angle Orthod. 2012;82(2):294-303

13. Yu IJ, Kook YA, Sung SJ, Lee KJ, Chun YS, Mo SS. Comparison of tooth displacement between buccal mini-implants and palatal plate anchorage for molar distalization: a finite element study. Eur J Orthod. 2011 Nov 2. [Epub ahead of print].
14. Upadhyay M, Yadav S, Nanda R. Vertical-dimension control during en-masse retraction with mini-implant anchorage. Am J Orthod Dentofacial Orthop. 2010;138(1):96-108.

15. Silva DL, Mattos CT, Sant'Anna EF, Ruellas AC, Elias CN. Cross-section dimensions and mechanical properties of esthetic orthodontic coated archwires. Am J Orthod Dentofacial Orthop. 2013:143(4 Suppl):S85-91.

16. Elayyan F, Silikas N, Bearn D. Mechanical properties of coated superelastic archwires in conventional and self-ligating orthodontic brackets. Am J Orthod Dentofacial Orthop. 2010;137(2):213-7.

17. Silva DL. Características dos fios ortodônticos estéticos Ortodontia e Odontopediatria [tese]. Rio de Janeiro (RJ): Universidade Federal do Rio de Janeiro; 2012

18. Oz AA, Arici N, Arici S. The clinical and laboratory effects of bracket type during canine distalization with sliding mechanics. Angle Orthod. 2012:82(2):326-32

19. Ong E, McCallum H, Griffin MP, Ho C. Efficiency of self-ligating vs conventionally ligated brackets during initial alignment. Am J Orthod Dentofacial Orthop. 2010;138(2):138.e1-7; discussion 138-139.

20. Nascimento LEAG. Bráquetes autoligados versus convencionais: aspectos microbiológicos e biomecânicos [tese]. Rio de Janeiro (RJ): Universidade Federal do Rio de Janeiro; 2013

21. Frye L, Diedrich PR, Kinzinger GS. Class II treatment with fixed functional orthodontic appliances before and after the pubertal growth peak - a cephalometric study to evaluate differential therapeutic effects. J Orofac Orthop. 2009:70(6):511-27.

22. Kinzinger GS, Savvaidis S, Gross U, Gülden N, Ludwig B, Lisson J. Effects of Class II treatment with a banded Herbst appliance on root lengths in the posterior dentition. Am J Orthod Dentofacial Orthop. 2011;139(4):465-9.

23. Bock NC, von Bremen J, Ruf S. Occlusal stability of adult Class II division 1 treatment with the Herbst appliance. Am J Orthod Dentofacial Orthop 2010;138(2):146-51

24. Bock NC, Ruf S. Class II division 2 treatment-does skeletal maturity influence success and stability? J Orofac Orthop. 2013;74(3):187-204

25. Greene CS. Relationship between occlusion and temporomandibular disorders: Implications for the orthodontist. Am J Orthod Dentofacial Orthop. 2011:139(1):11, 13, 15

\section{André Weissheimer}

» Degree in Dentistry, Federal University of Santa Catarina (UFSC).

»Specialist in Orthodontics, UFSC.

" $\mathrm{PhD}$ in Orthodontics, Catholic University of Rio Grande do Sul (PUCRS).

" $\mathrm{PhD}$, University of Southern California.

» Professor, Specialization course in Orthodontics, ABO-PA.

\section{Márlio Vinícius de Oliveira}

»Degree in Dentistry, School of Pharmacy and Dentistry of Alfenas, EFOA.

"Specialist in Orthodontics, EFOA.

» Certified by the Brazilian Board of Orthodontics and Facial Orthopedics (BBO).

\section{Matheus Melo Pithon}

»Adjunct professor, Department of Orthodontics, State University of Southwestern Bahia, UESB.

» $\mathrm{PhD}$ in Orthodontics, Federal University of Rio de Janeiro, UFRJ.

" Certified by the Brazilian Board of Orthodontics and Facial Orthopedics (BBO).

\section{Luiz Gonzaga Gandini Júnior}

" PhD in Orthodontics, State University of São Paulo, UNESP.

» PostDoc in Orthodontics, Baylor College of Dentistry.

" Full professor, state University of São Paulo, UNESP. 